\title{
Dissociating Self-Generated from Passively Applied Head Motion: Neural Mechanisms in the Vestibular Nuclei
}

\author{
Jefferson E. Roy and Kathleen E. Cullen \\ Aerospace Medical Research Unit, Department of Physiology, McGill University, Montreal, Quebec, Canada H3G 1Y6
}

\begin{abstract}
The ability to distinguish sensory inputs that are a consequence of our own actions from those that result from changes in the external world is essential for perceptual stability and accurate motor control. To accomplish this, it has been proposed that an internal prediction of the consequences of our actions is compared with the actual sensory input to cancel the resultant self-generated activation. Here, we provide evidence for this hypothesis at an early stage of processing in the vestibular system. Previous studies have shown that neurons in the vestibular nucleus, which receive direct inputs from vestibular afferent fibers, are responsive to passively applied head movements. However, these same neurons do not reliably encode head velocity resulting from self-generated movements of the head on the body. In this study, we examined the mechanism that underlies the selective elimination of vestibular sensitivity to active head-on-body rotations. Individual neurons were recorded in monkeys making active head movements. The correspondence between intended and actual head movement was experimentally controlled. We found that a cancellation signal was gated into the vestibular nuclei only in conditions in which the activation of neck proprioceptors matched that expected on the basis of the neck motor command. This finding suggests that vestibular signals that arise from self-generated head movements are inhibited by a mechanism that compares the internal prediction of the sensory consequences by the brain to the actual resultant sensory feedback. Because self-generated vestibular inputs are selectively cancelled early in processing, we propose that this gating is important for the computation of spatial orientation and control of posture by higher-order structures.
\end{abstract}

Key words: vestibular nucleus; self-motion; reafference; efference copy; gaze shift; vestibular reflexes; head-unrestrained

\section{Introduction}

To maintain perceptual stability, the nervous system must differentiate between sensory signals that register changes in the external world (exafference) and those that result from our own actions (reafference). Such a differentiation is also necessary for the construction of neural representations of the environment to accurately guide behavior. von Holst and Mittelstaedt (1950) originally proposed that the differentiation between exafference and reafference is ensured because the CNS sends a parallel "efference copy" of the motor command to sensory areas. In turn, this anticipatory signal is subtracted from the incoming sensory signal to selectively remove that portion caused by the animal's own actions. More recent studies have furthered this proposal by suggesting that an internal model of the sensory consequence of our actions, derived from motor efference copy, is compared with actual sensory inputs (Jeannerod 1988; Wolpert et al., 1995; Decety, 1996). Neurophysiological experiments in the electrosensory system of electric fish have provided support for this idea. During active electroreception, a negative image of the predicted reafference is generated and added to neurons at the first stage of central processing, resulting in the selective suppression of sen-

Received Aug. 28, 2003; revised Jan. 5, 2004; accepted Jan. 6, 2004.

This study was supported by the Canadian Institutes of Health Research and Fonds de la Recherche en Santé de Québec. We thank S. Sadeghi Ghandehari for assistance with some of the experiments. We also thank D. Guitton, D. Watt, P. A. Sylvestre, and S. Sadeghi Ghandehari for critically reading this manuscript and E. Moreau, W. Kucharski, J. Knowles, and A. Smith for excellent technical assistance.

Correspondence should be addressed to Kathleen E. Cullen, Aerospace Medical Research Unit, 3655 Promenade Sir William 0sler, Montreal, Quebec, Canada H3G 1Y6. E-mail: kathleen.cullen@mcgill.ca.

DOI:10.1523/JNEUROSCI.3988-03.2004

Copyright $\odot 2004$ Society for Neuroscience $\quad$ 0270-6474/04/242102-10\$15.00/0 sory information arising from self-generated currents (Bell et al., 1999; Bell, 2001).

The sensors of the vestibular system, like those of the electrosensory system, can be activated by self-generated as well as externally produced signals. Vestibular receptors and afferent fibers register information about the subject's own movement as well as movements that arise from changes in the external world (Cullen and Minor, 2002). However, a specific class of neurons in the vestibular nuclei, termed vestibular-only (VO) neurons, receive direct inputs from vestibular afferents but do not provide reliable information about head velocity during active rotations of the head on the body (Boyle et al., 1996; McCrea et al., 1999; Roy and Cullen, 2001). Remarkably, the same neurons continue to faithfully encode information about passive head rotations, which occur during the execution of voluntary movements.

Numerous extravestibular cues could function to attenuate the vestibular sensitivity of VO neurons during active head-onbody movements. For example, activation of neck proprioceptors influences the activity of vestibular nucleus neurons in decerebrate animals (Boyle and Pompeiano, 1981; Anastasopoulos and Mergner, 1982; Wilson et al., 1990; Wilson, 1991). However, passive activation of neck proprioceptors does not significantly affect processing in the vestibular nucleus in alert rhesus monkeys (Roy and Cullen, 2001). In addition, at least six cortical areas (for review, see Fukushima, 1997) send direct projections to the vestibular nuclei. In theory, these inputs could carry an inhibitory signal related to the monkey's knowledge of its head motion. However, in an experiment in which a rhesus monkey manually operated a steering wheel to control the movement of its body 
and head together in space, VO neurons continued to reliably encode head-in-space motion (Roy and Cullen, 2001).

Thus, the key question remains of how neuronal activity is selectively attenuated during active head-on-body movements. Here, we recorded from single neurons during passively applied movements and self-generated movements in which the correlation between intended and actual head movement was experimentally controlled. A cancellation signal was present only in conditions in which neck proprioceptive activation matched that expected from the neck motor command. Thus, our results provide support for the hypothesis that an internal model of the sensory consequences of active head motion is used to selectively suppress reafference at the level of the vestibular nuclei.

\section{Materials and Methods}

Four monkeys (3 Macaca mulatta and 1 Macaca fascicularis) were prepared for chronic extracellular recording using aseptic surgical techniques. All experimental protocols were approved by the McGill University Animal Care Committee and were in compliance with the guidelines of the Canadian Council on Animal Care.

\section{Surgical procedures}

The surgical techniques were similar to those previously described (Roy and Cullen, 2001). Briefly, a dental acrylic implant was attached to each animal's skull using stainless steel screws. Within the implant was embedded a stainless steel post that was used to restrain the animal's head during the experiment and two stainless steel recording chambers, which were positioned to provide bilateral access to the rostral-medial and ventral-lateral vestibular nuclei (posterior and lateral angles of $30^{\circ}$ ). In the same procedure, an 18- to 19-mm-diameter eye coil (three loops of Teflon-coated stainless steel wire) was implanted beneath the conjunctiva of the right eye. After the surgery, the animals were administered buprenorphine $(0.01 \mathrm{mg} / \mathrm{kg}$, i.m.) for postoperative analgesia and the antibiotic cefazolin $(25 \mathrm{mg} / \mathrm{kg}$, i.m., for $5 \mathrm{~d})$. Animals were given at least 2 weeks to recover from the surgery before experiments began.

\section{Data acquisition}

During the experiments, the monkey was comfortably seated in a primate chair that was fixed to the suprastructure of a vestibular turntable. The chair was placed in the experimental apparatus so that the animal's head was centered within a $1 \mathrm{~m}^{3}$ magnetic field coil system (CNC Engineering). Gaze and head positions were recorded using the magnetic search coil technique (Fuchs and Robinson, 1966). A specially designed head holder (Roy and Cullen, 2001) enabled us either to completely immobilize the animal's head (head-restrained) or to allow the animal full freedom of head motion (head-unrestrained). Monkeys were trained to track a small $\left(0.3^{\circ}\right.$ in diameter $)$ visual target for a juice reward. The target was generated by a HeNe laser and projected onto a white cylindrical screen located $60 \mathrm{~cm}$ from the monkey's eyes. The target was positioned on the screen by a pair of mirrors mounted on two computer-controlled galvanometers (General Scanning). Throughout the experiments, target contrast was $3 \log$ units above human perception, as measured by the method of Lisberger and Westbrook (1985).

REX, a QNX-based real-time data acquisition system (Hayes et al., 1982), was used to control target position, to monitor performance, and to collect data. Gaze, head, and target position signals were filtered (eight-pole Bessel, DC, $250 \mathrm{~Hz}$ ) and then digitized at $1000 \mathrm{~Hz}$. Turntable velocity was measured using an angular velocity sensor (Watson Inc.). Extracellular single-unit activity was recorded using enamel-insulated tungsten microelectrodes (7-10 M $\Omega$ impedance; Frederick-Haer). Horizontal and vertical gaze and head positions, target position, table velocity, and unit activity were recorded on digital audio tape for later playback. The isolation of each unit was carefully reevaluated off-line. During playback, action potentials were discriminated using a windowing circuit (BAK) that was manually set to generate a pulse coincident with the rising phase of each action potential.

\section{Experimental Design}

Recordings made in head-restrained monkeys. We confirmed that each neuron discharged in a manner consistent with previous characterizations of a subclass of neurons termed VO neurons (Scudder and Fuchs, 1992; Cullen and McCrea, 1993). Neurons were recorded while monkeys were passively rotated $(0.5 \mathrm{~Hz}, 40$ and $80 \% \mathrm{sec}$ peak velocity) about an earth vertical axis. Specifically, the head and body were rotated together en bloc, and data were collected during two specific paradigms. First, neurons were tested in response to rotations applied in the dark, during which monkeys generated compensatory eye movements in response to head rotation [i.e., the vestibulo-ocular reflex (VOR)]. Second, neurons were tested while monkeys fixated a target that moved with the vestibular turntable [i.e., the VOR cancellation paradigm (VORc)]. Moreover, each neuron was recorded while the monkey maintained ocular fixation at different horizontal and vertical eccentricities over a range of $\pm 20^{\circ}$ and during horizontal smooth pursuit $\left(0.5 \mathrm{~Hz}, 40\right.$ and $80^{\circ}$ /sec peak velocity).

With the animal's head still restrained, we then determined whether the presence of motor commands to the neck musculature altered the discharges of VO neurons in three conditions. First, neuronal responses during large saccadic eye movements were characterized by having the head-restrained monkey visually track a laser target that was stepped between horizontal positions over a range of $\pm 30^{\circ}$ or a food target, which appeared unexpectedly on any side of an opaque screen facing the monkey (Cullen and Guitton, 1997). The concurrent neck torque produced against the head restraint (reaction torque transducer; Sensotec) was recorded in three monkeys for a subset of neurons. Second, neural discharges were recorded while the monkey's head was held stationary relative to the earth, and its body was passively rotated at $0.5 \mathrm{~Hz}$ with a peak velocity of $80 \% \mathrm{sec}$ [body-under-head (BUH) rotation]. During BUH rotation, monkeys were again rewarded for making eye movements to laser and food targets, and the neck torque produced against the head restraint was measured. Third, neural discharges were recorded while the monkey's head and body were passively rotated together at $0.5 \mathrm{~Hz}$ with a peak velocity of $80^{\circ} / \mathrm{sec}$ as the monkey simultaneously generated neck torque (VOR + torque condition). As during BUH rotation, laser and food targets were presented to entice the monkeys to produce neck torque against the head restraint.

For the experiments in which monkeys were encouraged to generate neck torque, data were separately analyzed during (1) intervals in which torque was minimal $(<0.3 \mathrm{~N}-\mathrm{m})$ and (2) intervals in which torque reached high levels $(>1 \mathrm{~N}-\mathrm{m})$. To verify that these higher torque values were comparable with those generated by the monkey during natural head movements, we engaged the torque transducer by randomly braking the head movements just before large gaze shifts in head-unrestrained monkeys for a small subset of trials $(<5 \%)$. Torque was then measured while the monkey, unaware of the imposed restraint, attempted to move its head to generate a gaze shift.

Recordings made in head-unrestrained monkeys. After a neuron had been fully characterized in the head-restrained condition, the monkey's head was slowly and carefully released. With the head restraint freed, the monkey was able to rotate its head through the natural range of motion in the yaw (horizontal), pitch (vertical), and roll (torsional) axes. Neuronal activity was then recorded during two paradigms in which monkeys made voluntary combined eye-head movements. In the first paradigm, monkeys made natural voluntary combined eye-head movements of $15-65^{\circ}$ in amplitude (amplitude $=$ displacement of the eye-in-head + head-in-space motion) to orient to laser and food targets. These naturally generated gaze shifts served as controls and had velocities comparable with those used to determine the sensitivity to head motion during passive whole-body rotation (e.g., $<100 \%$ sec; Roy and Cullen, 2001). In the second paradigm, monkeys generated combined eye-head gaze shifts, and the velocity of their head-on-body motion was fed back into the vestibular turntable controller to simultaneously rotate the monkey in the opposite direction. Because the turntable rotated the monkey in the direction opposite to its ongoing head-on-body motion, head-in-space motion was reduced, whereas motion of the head relative to the body was not affected. The goal of this paradigm was to minimize the motion of the head relative to space; therefore, these gaze shifts were termed "reduced vestibular input” gaze shifts. A monkey's head movements were fed back 

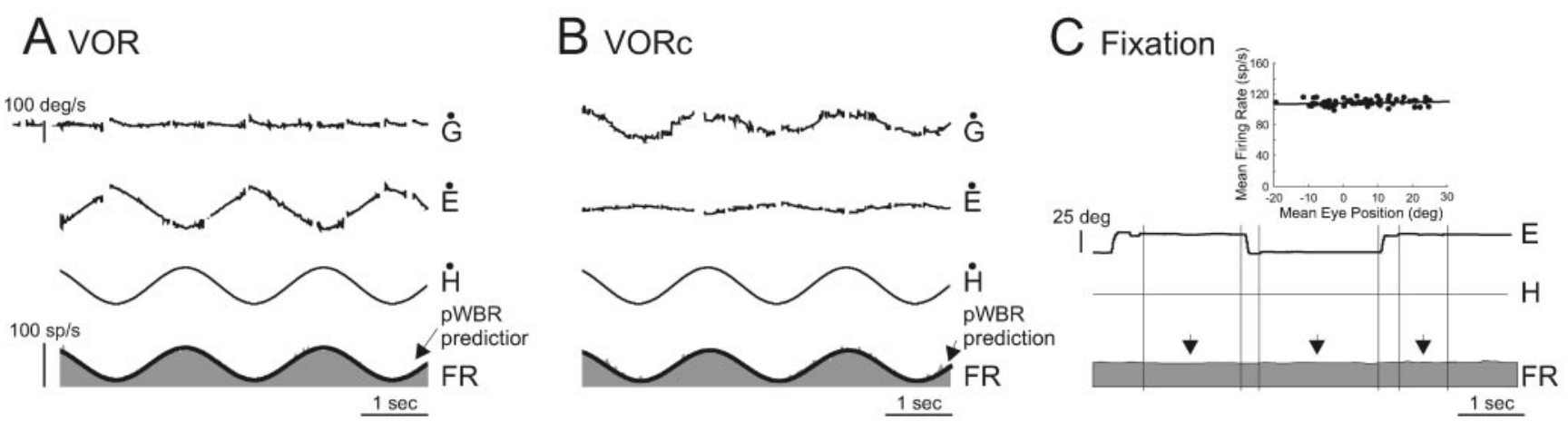

Figure 1. Activity of an example V0 neuron (unit b84_3) during the head-restrained condition. $A, B$, Passive whole-body rotation was used to characterize the response of the neuron to head movements during VOR in the dark $(A)$ and head movements while the monkey canceled its VOR by fixating a target that moved with the turntable $(B)$. A model based on head-restrained head movement sensitivities during VOR in the dark (pWBR prediction, thick trace) is superimposed on the firing rate traces. $C$, The neuron was unresponsive to changes in eye position during ocular fixation (vertical arrows). Inset, Regression of mean eye position and mean firing rate. Traces directed upward are in the ipsilateral direction. E, Eye position; $\mathrm{H}$, head position; $\mathrm{E}$, eye-in-head velocity; $\dot{H}$, head velocity; $\dot{G}$, gaze velocity $(=\dot{E}+\dot{H})$.

to drive the turntable servo controller on only a very small percentage of trials $(10 \%)$ to ensure that monkeys did not alter their natural gaze shift strategy. Moreover, the presentation of reduced vestibular input gaze shifts was randomized so that monkeys were unable to predict when their head movements would drive the turntable. To compensate for the delay $(\sim 15 \mathrm{msec})$ between active head movement and the resulting turntable motion, the controller gain was set to 1.5 for the initial acceleration phase of the active head movement $(\sim 100 \mathrm{msec})$.

During reduced vestibular input gaze shifts, we successfully maintained isolation of, in three monkeys, a total of eight cells in the medial vestibular nucleus that we classified as VO neurons. To get sufficient data for the analysis of neuronal responses during reduced vestibular input gaze shifts, it was necessary to first identify a neuron on the basis of the criteria that conformed to previous descriptions of VO neurons in a head-restrained monkey (see Fig. $1 A-C$ ), to preserve isolation after the transition from head-restrained to head-unrestrained recording, to collect control data during normal gaze shifts, and to finally maintain isolation during the reduced vestibular input condition. Maintaining isolation of neurons during voluntary gaze shifts in the reduced vestibular input experiment was particularly challenging because monkeys were unsettled by this paradigm; they frequently generated high-frequency shaking head movements after each trial, which in turn produced small displacements of the brainstem relative to the microelectrode tip. Thus, it was considerably more difficult to maintain neuronal isolation during this paradigm than during control gaze shifts.

\section{Analysis of neuron discharges}

Data were imported into the Matlab (The MathWorks, Natick, MA) programming environment for analysis. Recorded gaze and head position signals were digitally filtered with zero phase at $125 \mathrm{~Hz}$ using a 51st-order finite impulse response filter with a Hamming window. Eye position was calculated from the difference between gaze and head position signals. Gaze, eye, and head position signals were digitally differentiated to produce velocity signals. The neural discharge was represented using a spike density function in which a Gaussian was convolved with the spike train (SD of $5 \mathrm{msec}$ for saccades and gaze shifts and $10 \mathrm{msec}$ for the remaining paradigms; Cullen et al., 1996). Saccade and gaze shift onsets and offsets were defined using a $\pm 20 \% \mathrm{sec}$ gaze velocity criterion. Statistical significance was determined using paired Student's $t$ tests. A least squared regression analysis was used to determine the resting discharge (bias, spikes per second), head velocity sensitivity [ $g$, (spikes per second)/(degrees per second)]), and the phase shift of each neuron relative to head velocity during passive whole-body rotation, passive headon-body rotations, and control gaze shifts.

\section{Results}

All neurons included in the present report $(n=52)$ were classified as VO neurons on the basis of their response patterns in the head-restrained condition. Depending on whether their activity increased during ipsilaterally $(n=33)$ or contralaterally $(n=19)$ directed passive whole-body rotation, neurons were further classified as type I or II, respectively. When the monkey was passively rotated about an earth vertical axis in the dark, each neuron was modulated in response to head velocity (Fig. 1A). Because passive rotation in the dark elicits a compensatory eye motion response (i.e., the VOR), neurons were also characterized during passive whole-body rotation while the monkey cancelled its VOR (VORc) by fixating a visual target that moved with its head (Fig. $1 B$ ). Neuronal head velocity sensitivities during VOR and VORc were comparable [sample mean head velocity sensitivity \pm SEM, $0.54 \pm 0.04$ and $0.53 \pm 0.04$ ( spikes $/ \mathrm{sec}) /(\% \mathrm{sec})$, respectively; $p>$ $0.5]$. Moreover, all neurons were unresponsive to eye position during ocular fixation and relatively small $\left(<20^{\circ}\right)$ saccades (Fig. $1 C$ ) and during smooth pursuit (data not shown). Thus, during head-restrained eye movements and passive whole-body rotations, each neuron responded in a manner consistent with previous characterizations of VO neurons (Fuchs and Kimm, 1975; Keller and Kamath, 1975; Tomlinson and Robinson, 1984; Scudder and Fuchs, 1992; Cullen and McCrea, 1993; McCrea et al., 1999; Roy and Cullen, 2001). For the purpose of this paper, type I and II neurons were considered collectively because they encoded similar signals during each behavioral task.

A series of experiments were then done to distinguish between four possible mechanisms for the reduction of vestibular sensitivity during active head rotations (Fig. 2). First, it is possible that an internal model of the motor command to the neck musculature (e.g., neck efference copy signal) provides direct inhibitory inputs to VO neurons (mechanism A). Alternatively, during selfgenerated head-on-body motion, an internal model of the command to move the head could be used to gate in inhibitory neck proprioceptive signals (mechanism B) or to selectively gate out vestibular-related modulation on VO neurons (mechanism C). Finally, it is possible that an inhibitory neck proprioceptive signal is gated in only when the actual activation of neck proprioceptors matches an internal model of the consequence of head motion (mechanism D).

\section{Test of hypothesis A: do neck motor commands attenuate VO responses?}

We first tested whether neck efference copy signals provide direct inhibitory inputs to VO neurons (mechanism A; Fig. 2) by spe- 


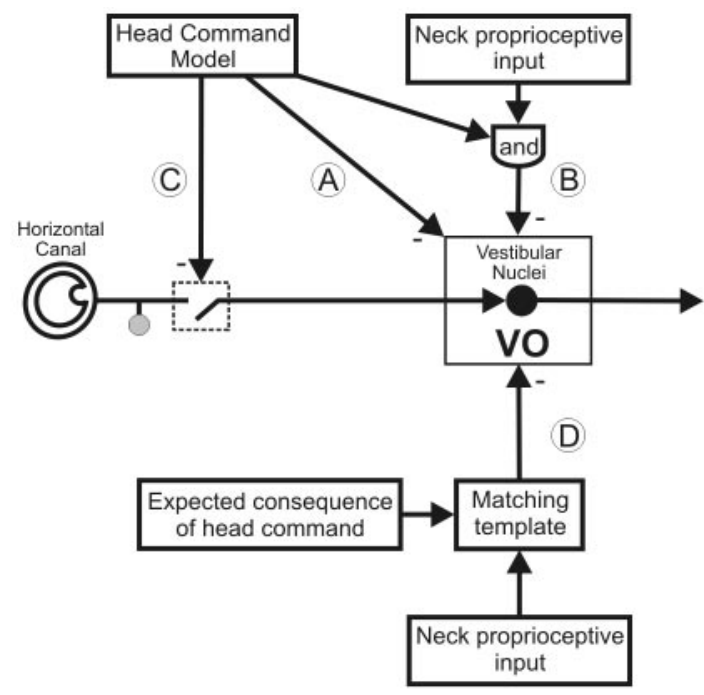

Figure 2. Four possible mechanisms that could be responsible for the observed attenuation of VO neurons during self-generated head-on-body motion. See Results for details.

cifically characterizing their response during large-amplitude $\left(>25^{\circ}\right)$ saccades. Large saccades in monkeys (Bizzi et al., 1971; Vidal et al., 1982; Lestienne et al., 1984) and humans (AndréDeshays et al., 1991) are normally accompanied by the production of significant neck torque even if the subject is headrestrained. However, previous studies have not described the responses of VO neurons during large head-restrained saccades. To confirm that large saccades were accompanied by the generation of neck torque and, in turn, to test whether the underlying neck motor command provided direct inhibitory inputs to VO neurons, we measured the neck torque produced by the monkey during large-amplitude saccades for a subset of neurons $(n=12)$ in three monkeys.

In Figure 3, eye and torque trajectories and unit responses of example neuron cr37_3 were aligned on saccade onset for ipsilaterally (left plots) and contralaterally (right plots) directed saccades (re: the directional preference of its head velocity sensitivity during VOR), respectively. Figure $3, A$ and $B$, illustrates the neural responses during saccades accompanied by the generation of relatively low $(<0.3 \mathrm{~N}-\mathrm{m})$ versus high $(>1 \mathrm{~N}-\mathrm{m})$ neck torques, respectively. During saccades accompanied by higher torques, the firing rate of the neuron remained constant and unchanged from its responses during either ocular fixation or lower-torque saccades. In Figure $3 C$, eye and torque trajectories and unit response were aligned on torque onset for the set of saccades that were accompanied by higher torque. Again, during highertorque saccades, the activity of the neuron remained constant and unchanged from its responses during either fixation or lowertorque saccades. Similar results were obtained for each neuron in our sample; there was no significant difference between neuronal firing rate during individual large saccades accompanied by torque and firing rate during intervals of ocular fixation ( $p$ range, $0.25-0.75$, unpaired $t$ test). Accordingly, the mean discharge calculated across neurons was $69 \pm 16$ spikes/sec during fixation, which was not significantly different $(p>0.05)$ from that measured during either ipsilaterally or contralaterally directed saccades $(64 \pm 14$ and $67 \pm 15$ spikes/sec, respectively; Fig. $4 \mathrm{~A}$, inset).

The plot in Figure $4 A$ compares, for each neuron, mean firing rates during large saccades that were accompanied by large torques and mean firing rates during ocular fixation. If the com- mand to move the head produced an inhibitory drive to $\mathrm{VO}$ neurons that reduced their response to vestibular stimulation during gaze shifts (mechanism A; Fig. 2), then the background discharge rate of VO neurons should have been reduced for large saccades when the head movement command was issued because the vestibular stimulation was prevented by the head restraint. However, this was not the case. The regression equation relating these data was saccades firing rate $\left(F R_{\text {saccades }}\right)=2+0.93$ * $F R_{\text {fixation, }}$, and the slope of the regression line did not differ from unity $(p>0.05)$, confirming that neuronal responses remained constant and unchanged from ocular fixation. Moreover, we verified that the torques generated during large head-restrained saccades were comparable with those generated during natural gaze shifts by momentarily engaging the torque transducer just before gaze shift onset (Fig. 4A, bottom right inset). Using this method, torques in the range of $0.2-1.5 \mathrm{~N}$-m were typically recorded. Thus, taken together, these results are not consistent with the hypothesis that a neck efference copy signal provides direct inhibitory inputs to $\mathrm{VO}$ neurons to suppress vestibular reafference (mechanism A; Fig. 2).

For the remaining neurons $(n=40)$, we recorded neither torque nor neck EMG during head-restrained saccades. For these neurons, we inferred that the monkey generated active head motor commands in addition to the observed saccadic eye movements. We thought this assumption was safe because of our observation in the subset of neurons (Figs. 3, 4A) that most large saccades were accompanied by the production of neck torque and also because this property has previously been well established in monkeys and humans (see above references). The findings were comparable with those shown in Figures 3 and $4 A$. Specifically, during large ipsilateral and contralateral saccades, the firing rate of each neuron remained constant and unchanged from that observed during ocular fixation ( $p$ range, $0.40-0.73$, unpaired $t$ test). Accordingly for the sample of neurons (Fig. $4 B$, top left inset), mean firing rates did not differ for either ipsilaterally or contralaterally directed saccades $(58 \pm 5$ and $61 \pm 6$ spikes/sec, respectively) and ocular fixation (58 \pm 5 spikes/ sec; paired $t$ test, $p>0.05$ and 0.05 , respectively). A comparison of the mean firing rate of each neuron during large saccades and ocular fixation is plotted in Figure $4 B$. The regression equation relating these data was $F R_{\text {saccades }}=1+1.00{ }^{*} F R_{\text {fixation, }}$, where again as in Figure $4 A$, the regression slope did not differ significantly from unity $(p>0.05)$.

\section{Test of hypothesis B: do head motor commands gate inhibitory neck proprioceptive inputs?}

We next investigated whether an internal model of the command to move the head might gate in existing neck proprioceptive signals (mechanism B; Fig. 2) to inhibit VO neurons during active head-on-body movements. During passive rotation of the body under a stationary head, the vestibular system is not activated, but, instead, neck proprioceptors are activated by the stretching of the neck musculature (e.g., Richmond and Abrahams, 1979; Kasper et al., 1989). Accordingly, VO neurons were recorded while the monkey's body was passively rotated beneath its earthstationary head and the monkey simultaneously generated neck torque to orient to targets (see Materials and Methods). The responses of two example neurons, cr37_3 (same as in Fig. 3) and cr42_2, are illustrated in Figure 5, $A$ and $B$, respectively. The example traces are typical in that the activity of each neuron remained unchanged from that observed during ocular fixation regardless of whether the monkey tried to move its head in the ipsilateral or contralateral direction during passively applied body rotation (compare the time intervals denoted between the 
A Low torque

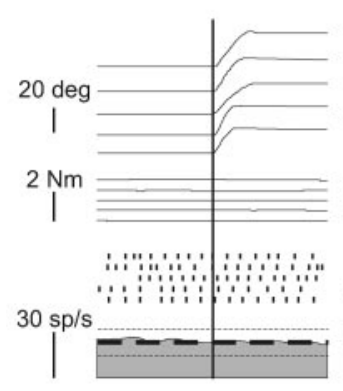

B High torque

Aligned on eye onset

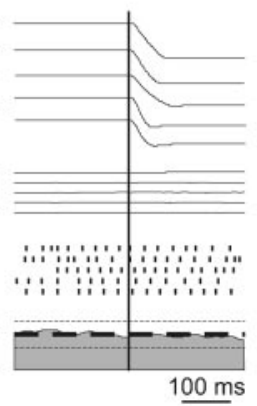

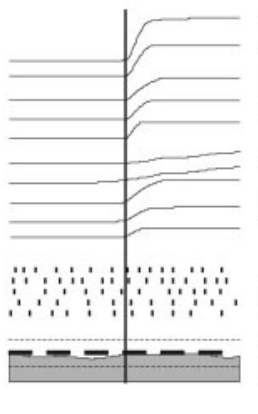

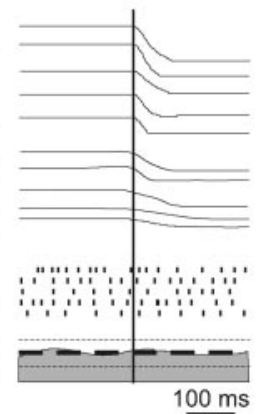

C High torque

Aligned on torque onset

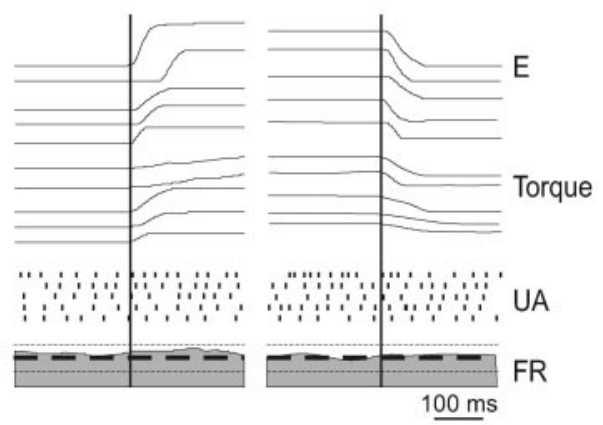

Figure 3. Activity of example V0 neuron cr37_3 during head-restrained saccades. $A, B$, The example neuron was unresponsive during ipsilaterally (left plots) and contralaterally (right plots) directed saccades when the monkey produced little torque $(A,<0.3 \mathrm{~N}$-m). Similarly, the response of the neuron was not changed when the monkey generated relatively higher levels of torque ( $B$, $>1 \mathrm{~N}-\mathrm{m})$. Traces are aligned on the start of the saccades. C, Saccades from $B$ with the traces now aligned on torque onset. Individual action potentials, represented by tick marks, are shown below the eye traces (UA). The mean firing rate $\pm S D$ is plotted on the firing rate (bottom trace).

two sets of vertical lines for each neuron). Note that during intervals of passive body rotation in which the monkey was not attempting to move its head, torque remained near zero. Regression analysis verified that neuronal discharges were not systemically modulated as a function of neck torque in either direction (Fig. 5A,B, insets; slope $=0 ; p>0.05$ ).

Similar results were obtained for all VO neurons $(n=8)$ tested. As shown in Figure $5 C$, the slope of the regression line comparing mean firing rate during fixation and mean firing rate during $\mathrm{BUH}$ with torque did not differ significantly from unity $(p>0.05)$. Firing rate discharges were comparable during both contralateral and ipsilateral torque conditions (Fig. $5 C$, inset; sample mean firing rates, $74 \pm$ 22 and $72 \pm 20$ spikes/sec; gray, filled columns, respectively; $p>0.5$ ) and were not significantly different from those measured during ocular fixation (Fig. $5 C$, open column; sample mean firing rate, $77 \pm 22$ spikes/sec; $p>0.05$ ). Moreover, no individual neuron showed a significant difference in discharge rate during ipsilaterally or contralaterally directed torques and ocular fixation ( $p$ range, $0.35-0.62$ ).

\section{Test of hypothesis C: do head motor commands selectively} gate out vestibular inputs?

We next examined the possibility that an internal model of the command to move the head might selectively gate out vestibularrelated modulation on $\mathrm{VO}$ neurons to reduce their sensitivity to vestibular input during active head-on-body movements (mechanism C; Fig. 2). Head-restrained monkeys underwent passive whole-body rotations during which they were encouraged to produce neck torque by orienting to food targets. Because the head was restrained in this paradigm, the pattern of neck proprioceptor activation differed from the pattern that would have occurred had the same neck motor command been generated with the head unrestrained. The responses of the same example neurons shown in Figure 5, $A$ and $B$, are illustrated in Figure 6, $A$ and $B$, respec-

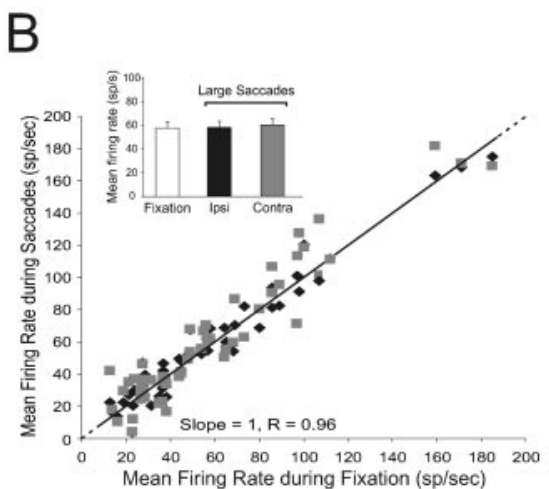

Figure 4. Summary of V0 neuron responses during large saccades. $A$, The mean firing rate of individual V0 neurons was comparable during ocular fixation and large ipsilaterally (Ipsi, solid diamond) and contralaterally (Contra, gray squares) directed Sample mean firing rates were not significantly different during fixation (open column) and during large ipsilaterally (filled column) and contralaterally (gray column) directed saccades. Dashed lines indicate unity (slope $=1$ ).

tively. Neuronal sensitivities to head velocity and bias discharge rates were comparable during head rotations, which were accompanied by little or no neck torque $(<0.3 \mathrm{~N}-\mathrm{m})$ and higher levels of torque $(>1.0 \mathrm{~N}-\mathrm{m})$. This is illustrated in Figure $6 \mathrm{~A}$ (compare left, right sides of traces), where the vestibularly driven modulation is constant regardless of the amount of torque that is concurrently produced by the monkey. Analogous results were obtained for all VO neurons $(n=8)$ tested; as shown in Figure $6 C$, the slope of the regression did not differ significantly from unity $(p>0.05)$. Overall, head velocity responses were comparable during contralateral and ipsilateral torque conditions [Fig. $6 \mathrm{C}$, inset; mean head velocity sensitivity, $0.58 \pm 0.17$ and $0.58 \pm 0.17$ (spikes/ $\mathrm{sec}) /(\% \mathrm{sec})$; gray, filled columns, respectively; $p>0.05$ ] and were not significantly different from those measured during VOR [Fig. $6 C$, open column; mean head velocity sensitivity, $0.59 \pm 0.17$ (spikes/sec) $/(\% / \mathrm{sec}) ; p>0.05]$. Moreover, as during headrestrained saccades and passive rotation of the body under the head, individual neurons showed no significant difference in discharge rates during ipsilaterally or contralaterally directed torque and their discharge rates during intervals of VOR ( $p$ range, 0.35 

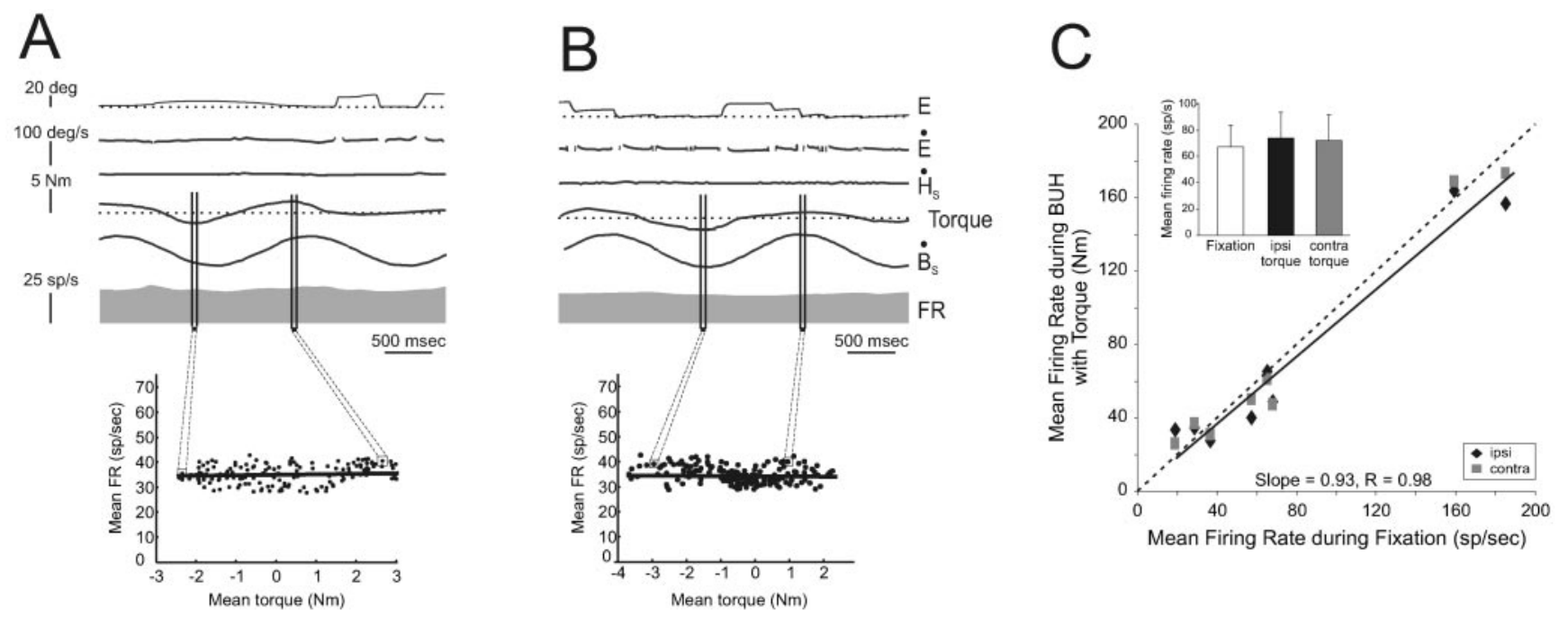

Figure 5. Activity of two example V0 neurons during passive body-under-head rotation. $A, B$, Example neurons $\mathrm{cr37} 3(A)$ and $\mathrm{cr} 42 \_2(B)$ were typical in that they were not modulated when the monkey's body was passively rotated beneath its earth-stationary head even when the monkey generated neck torque. C, The mean firing rate of each neuron was comparable during ocular fixation and passive neck rotation accompanied by the generation of neck torque in either the ipsilateral (Ipsi, solid diamonds) or contralateral (Contra, gray squares) direction. Inset, Average mean firing rates were not significantly different during ocular fixation (open column) and when ipsilaterally (filled column) or contralaterally (gray column) directed torque was generated. Dashed line indicates unity (slope $=1$ ).
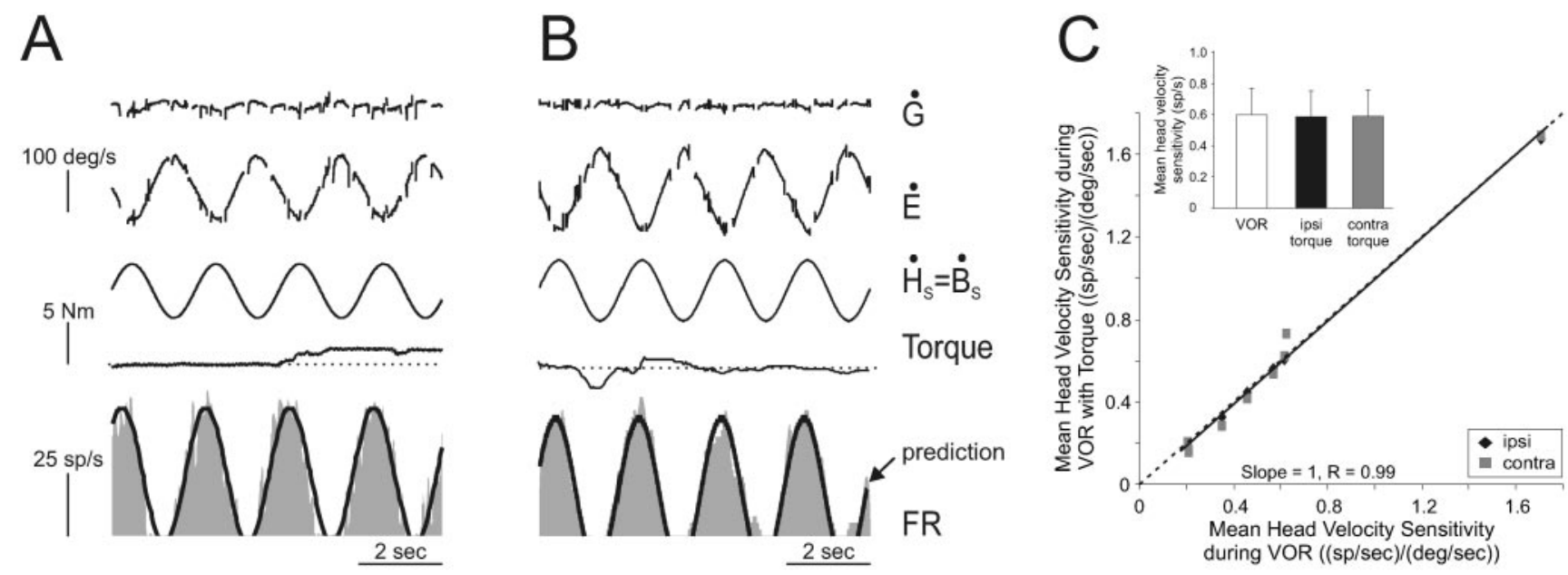

Figure 6. Activity of two example V0 neurons during intervals of passive whole-body rotation in which monkeys generated neck torque. $A, B$, Example neurons $c r 37 \_3(A)$ and cr42_2(B) were typical in that their responses to head velocity were comparable regardless of whether the monkey generated neck torque during passive whole-body rotation. A model based on neuronal responses to head velocity during VOR during periods when no torque was produced continued to describe the neuronal modulations during intervals in which torque was produced (prediction, thick trace). C, The mean head velocity sensitivity of each neuron was comparable during the VOR when no torque was produced and when torque was generated. Inset, Average head velocity sensitivities were not significantly different during VOR without torque (open column) and when either ipsilaterally (Ipsi, filled column) or contralaterally (Contra, gray column) directed torque was generated during passive whole-body rotation. Dashed line indicates unity $($ slope $=1)$.

0.77 and 0.32-0.69, head velocity sensitivity and bias, respectively).

\section{Test of hypothesis D: VO inhibition determined by matching expected and actual neck proprioceptive input}

Previous studies have shown that the head movement sensitivity of VO neurons is greatly attenuated during active eye-head gaze shifts (McCrea et al., 1999; Roy and Cullen, 2001). We verified this property in the present sample. Figure $7 A$ shows an example neuron during gaze shifts; the attenuation in head velocity sensitivity of the neuron is evident when the neuronal discharge (gray trace) is compared with superimposed prediction based on its response during passive whole-body rotations (VOR prediction, filled thick trace). During active combined eye-head gaze shifts, the head movement sensitivity our sample of VO neurons $(n=$ $45)$ was greatly attenuated relative to its sensitivity to head motion during passive whole-body rotation ( $65.8 \pm 4 \%$ attenuation; $p<0.05)$.

At first glance, the results shown above in Figures 3-6 appear to suggest that signals related to neck motor commands do not play an important role in mediating this inhibition. However, it is important to note that a common feature of each of these tasks was that the monkey generated neck motor commands, but the movement of the head relative to the neck was determined experimentally. Accordingly, we next tested whether an inhibitory neck proprioceptive signal might be gated in only when the actual activation of neck proprioceptors matches an internal model of the sensory consequence of head motion (mechanism D; Fig. 2). 


\section{A Control Gaze Shifts}

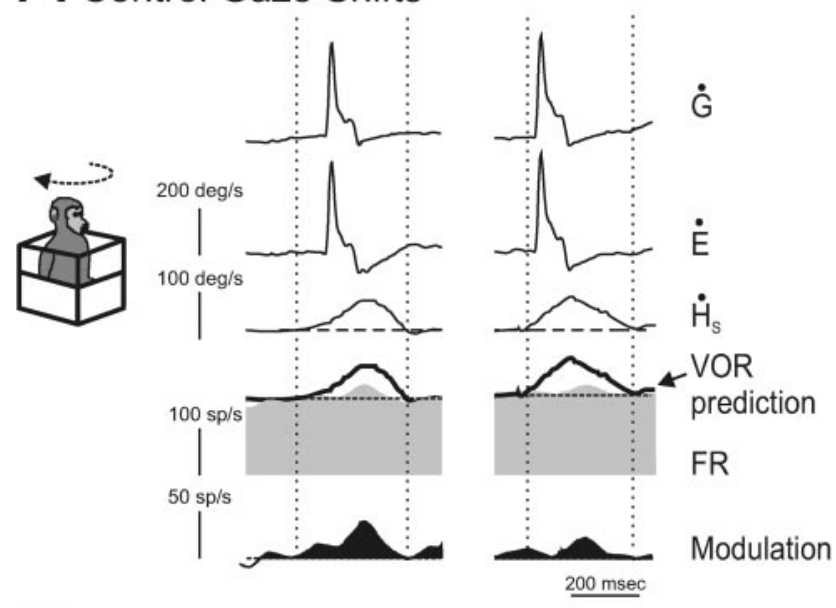

B Reduced Vestibular Input Gaze shifts
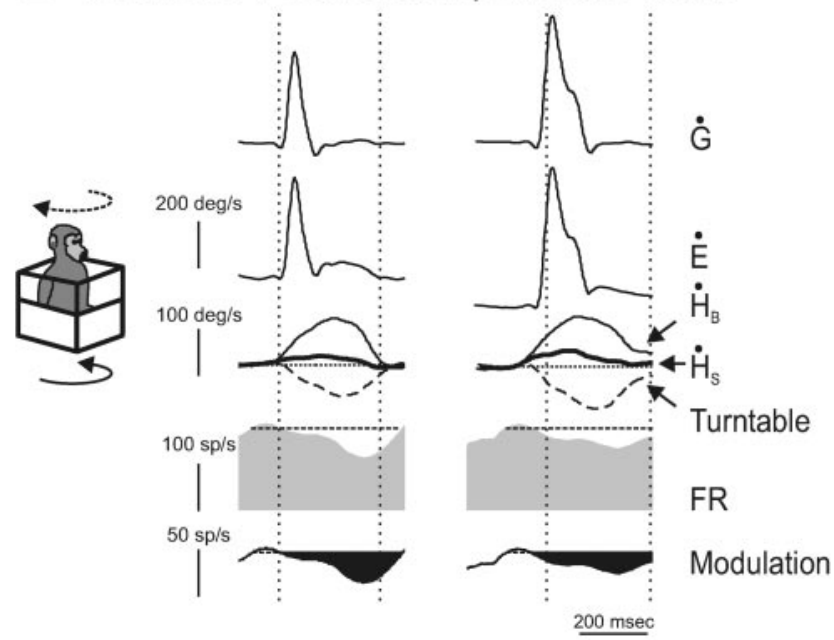

Figure 7. Activity of an example neuron during active head-on-body movements. $A$, Responses of neuron $\mathrm{cr} 122$ _3 during coordinated eye and head gaze shifts (active head movement is dashed line arrow in schema). The mean discharge rate during ocular fixation is superimposed on the firing rate (dashed line, second row from bottom). In addition, a prediction of the response of the neuron based on it response to passive whole-body rotation is superimposed (VOR prediction, thick trace). The black filled trace (bottom row) represents the difference between the mean firing rate during ocular fixation and gaze shifts. Note that the scale has changed. $B$, Response of the same neuron during the reduced vestibular input gaze shift paradigm. In this paradigm, the head motion actively generated by the monkey (dashed line arrow in schema) was fed to the vestibular turntable controller so that the whole monkey was simultaneously rotated in the opposite direction (solid arrow in schema). The resultant head-in-space motion $\left(\dot{H}_{\mathrm{S}}=\dot{H}_{\mathrm{B}}+\right.$ turntable; thick trace) was significantly reduced from what it would had been during a control gaze shift, but the movement of the head relative to the body $\left(\dot{H}_{B}\right)$ was not affected. The mean discharge of the neuron during ocular fixation is superimposed on the firing rate (dashed line, second row from bottom). The black filled trace (bottom row) represents the difference between the mean firing rate during ocular fixation and reduced vestibular input gaze shifts. Note that the scale has changed.

To do this, we recorded from VO neurons during voluntary eyehead gaze shifts during which the head-in-space motion generated by the monkey (Fig. $7 B$, dashed line arrow in schema) was minimized by rotating the table in the opposite direction. Specifically, head-in-space motion was minimized by feeding an inverted replica of the monkey's current head velocity into the turntable controller (Fig. 7B, black arrow in schema). As a consequence, head-in-space $\left(\dot{H}_{\mathrm{S}}\right)$ motion was significantly reduced from what it would have been during control gaze shifts (Fig. $7 B$,
$\dot{H}_{\mathrm{S}}$, thick trace), but the movement of the head relative to the body $\left(\dot{H}_{\mathrm{B}}\right.$, thin trace) was minimally affected. Thus, motor efference copy and resultant neck proprioceptive activation were matched as during control gaze shifts; however, the activation of the vestibular sensors was significantly reduced.

During these reduced vestibular input gaze shifts, all neurons $(n=8)$ showed a clear inhibition relative to control gaze shifts and, importantly, relative to the mean discharge during ocular fixation. This is shown for the example neuron (compare unit activity in Fig. 7A,B). The inhibition spanned the duration of the active head-on-body motion such that it included not only the duration of the gaze shift but also the period that immediately followed in which the gaze was stable in space but the head was still moving. This result suggests that the inhibition recorded during gaze shifts with reduced vestibular input could account for the attenuation of $\mathrm{VO}$ neuronal responses that is observed during gaze shifts. To pursue this argument further, we used the following analysis.

We hypothesized that the modulation of neurons during all paradigms should be described by the summation of their vestibular inputs and an inhibitory signal arising from active rotations of the head relative to the body:

$$
F R=\text { bias }+g_{\mathrm{VOR}} \times \dot{H}_{\mathrm{s}}-g_{\text {active }} \times \dot{H}_{\mathrm{B}(\text { active })},
$$

where $g_{\mathrm{VOR}}$ is the vestibular sensitivity to head velocity, and $\dot{H}_{\mathrm{B}(\mathrm{ac}}$ tive) is the self-generated motion of the head relative to the body. The vestibular-only gain, $g_{V O R}$, can be calculated from the response of each neuron during VOR because there are no active head movements in this condition.

Accordingly, during VOR generated by whole-body rotation, because $\dot{H}_{\mathrm{B}(\text { active })}=0$, Equation 1 simplifies to:

$$
F R_{\mathrm{VOR}}=\text { bias }+g_{\mathrm{VOR}} \times \dot{H}_{s} .
$$

During control gaze shifts, because $\dot{H}_{\mathrm{B}(\text { active })}=\dot{H}_{\mathrm{S}}$, Equation 1 becomes:

$$
F R_{\mathrm{gs}}=\text { bias }+g_{\mathrm{VOR}} \times \dot{H}_{\mathrm{s}}-g_{\text {active }} \times \dot{H}_{\mathrm{s}} .
$$

Accordingly, if the sensitivity of a neuron to $\dot{H}_{\mathrm{S}}$ during active gaze shifts is $g_{\mathrm{gs}}$, such that:

$$
F R_{\mathrm{gs}}=\text { bias }+g_{\mathrm{gs}} \times \dot{H}_{\mathrm{s}}
$$

it follows that:

$$
g_{\text {active }}=g_{\mathrm{VOR}}-g_{\mathrm{gs}} \text {. }
$$

Thus, the neuronal responses during reduced vestibular input gaze shifts should be predicted on the basis of the difference between responses to $\dot{H}_{\mathrm{S}}$ during passive whole-body rotation (VOR) and control gaze shifts. We verified whether this was the case. Figure $8 \mathrm{~A}$ (top plot) illustrates our approach for the example neuron shown in Figure 7. First, we optimized the parameters in Equations 2 and 3 in different experiments to fit the responses of the neuron during VOR $\left[g_{\mathrm{VOR}}=1.1(\mathrm{spikes} / \mathrm{sec}) /(\% \mathrm{sec})\right]$ and gaze shifts $\left[g_{\mathrm{gs}}=0.24\right.$ ( spikes $\left.\left./ \mathrm{sec}\right) /(\% \mathrm{sec})\right]$, respectively. Then the coefficient of the inhibition term $\left(g_{\text {active }}\right)$ was calculated using Equation $4\left[g_{\text {active }}=1.1-0.24=0.86(\right.$ spikes $\left./ \mathrm{sec}) /(\% \mathrm{sec})\right]$. Next, the coefficient of inhibition was obtained from Equation 1 by optimization using data from the reduced vestibular input gaze shift condition. The coefficient obtained directly from Equation 1 (estimated) was identical to that predicted by Equation 4 [e.g., 0.86 vs 0.86 (spikes/sec)/(deg/sec)]. This result is illustrated graphically in the bottom plot of Figure $8 \mathrm{~A}$. The FR prediction 


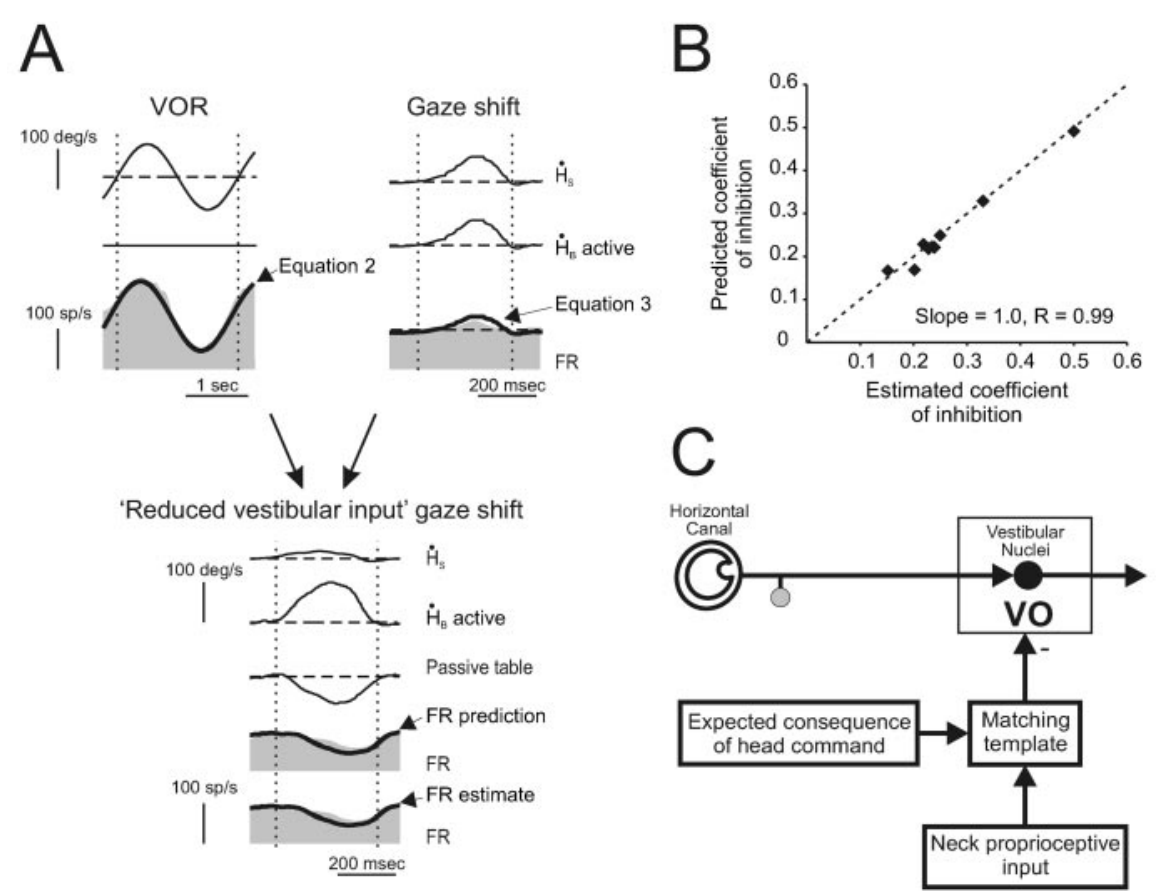

Figure 8. The reduction in modulation during the reduced vestibular input gaze shifts could be predicted on the basis of the difference between responses to $\dot{\mathrm{H}}_{\mathrm{S}}$ during passive whole-body rotation (VOR) and control gaze shifts. $A$, This neuron was typical in that the inhibition that occurred during reduced vestibular input gaze shifts (FR estimate) was identical to that predicted (FR prediction) on the basis of the same neurons response in two different experiments: VOR (Equation 2) and control gaze shifts (Equation 3). B, For each neuron in the sample tested, the estimated coefficient of inhibition during reduced vestibular input gaze shifts was comparable with the inhibition predicted by neural responses during VOR and control gaze shifts. C, Schematic to explain the present findings. A cancellation signal is gated into the vestibular nuclei only in conditions in which the activation of neck proprioceptors matched that expected on the basis of the neck motor command.

trace was obtained by substituting into Equation 1 the value of $g_{\text {active }}$ calculated from Equation 4 and predicting the FR during the reduced vestibular input gaze shift using the monkey's actual $\dot{H}_{\mathrm{B}}$ and $\dot{H}_{\mathrm{S}}$ as inputs. The FR estimate solid line was obtained by optimization using Equation 1.

The example neuron was typical in that this approach could be used to predict neuronal responses recorded during reduced vestibular input gaze shifts. Overall, for our sample of neurons, the estimated and predicted coefficients of inhibition were comparable for reduced vestibular input gaze shifts (Fig. $8 B$; mean inhibition, slope $=1.0 ; p>0.05)$. We therefore conclude that Equation 1 provides a valid description of neurons during active head-on-body movements under the conditions in the present experiments, and the inhibition recorded during reduced vestibular input gaze shifts is consistent with the attenuation of VO neuronal responses during gaze shifts.

\section{Discussion}

Neurons in the vestibular nucleus, which receive direct inputs from the vestibular afferents, are responsive to head velocity during passive whole-body rotations or passive head-on-body movements (Boyle et al., 1996; McCrea et al., 1999; Roy and Cullen, 2001). However, these same neurons do not provide reliable information during active movements of the head on the body. Here the mechanism that underlies this selective elimination of vestibular sensitivity to active head-on-body movements was investigated. We found that a cancellation signal is sent to the vestibular nuclei in conditions in which neck proprioceptive inputs match those expected on the basis of the neck motor command but not when these inputs are vastly different.

\section{The vestibular nuclei and the principle of reafference}

It had been postulated that an efference copy of the motor command to move the head could cancel vestibularly driven responses in the vestibular nuclei during active head-on-body motion (Boyle et al., 1996; McCrea et al., 1999; Roy and Cullen, 2001), consistent with the original theory of von Holst and Mittelstaedt (1950). In the present study, VO neurons were recorded in conditions in which neck motor commands were generated, but the resultant neck proprioceptive and vestibular feedback were determined experimentally. Neurons were first recorded while monkeys made large saccades but were not permitted to move their heads. During large head-restrained saccades, neck activation is strongly coupled with eye movements [human (André-Deshays et al., 1991) and monkey (Bizzi et al., 1971; Lestienne et al., 1984)]. In addition, we verified that the intensity of the head movement command was comparable with that issued during head-unrestrained gaze shifts. Nevertheless, we never detected an inhibitory drive to vestibular nuclei neurons. The largest torques that we measured in the headrestrained condition were on the order of 3.5-4 N-m, which compare well with the maximal rotational torques that can be generated by healthy male human subjects in the horizontal plane $(4.5 \mathrm{~N}-\mathrm{m}$; Tangorra et al., 2003). Thus, overall, monkeys appeared to remain optimistic about their ability to potentially make head movements.

There is an essential difference between head-restrained saccades and head-unrestrained gaze shifts. In the headunrestrained condition, the neck musculature is physically stretched and, in turn, the muscle spindles and Golgi tendon organs that innervate the neck musculature are activated (Richmond et al., 1988). In contrast, during head-restrained saccades, although neck proprioceptors are also activated (because the drive to muscles will activate $\gamma$ reflex pathways), the overall level of activation is reduced, and the dynamics of the activation differs significantly from what would have occurred if the head had been free to move (Vallbo, 1981). Thus, when a head movement command is issued during head-restrained saccades, the resultant neck proprioceptor activation does not match the activation that would be expected by the same motor command had the head been allowed to move.

Vestibular nuclei neurons are responsive to passive neck proprioceptor activation in decerebrate animals (Boyle and Pompeiano, 1981; Anastasopoulos and Mergner, 1982; Wilson, 1991). In contrast, in the alert rhesus monkey, passive activation of neck proprioceptors does not influence VO neuron activity (Roy and Cullen, 2001). One possible explanation for this apparent discrepancy is that the neck proprioceptive inputs to the vestibular nuclei are "gated out" in alert animals. Indeed, we found that an inhibitory input was only evident when the activation of neck proprioceptors corresponded to that expected as a result of the head movement command (mechanism D; Fig. 2). Because of the 
difficulty of the experimental design, our sample size was small; nevertheless, our results were uniformly consistent across the neurons tested.

\section{Sensory-motor matching and the gating of reafference}

The selective gating of proprioceptive inputs could be derived from the actual motor command to the neck musculature. Alternatively, an internal model of the head plant could be used. The distinction is an important one because in the head-restrained condition, a monkey presumably has knowledge of the head restraint, which could be used in the computation of the internal plant model. Thus, a schema that incorporates an internal model of the head plant would also be consistent with our results that an inhibitory signal is not observed when the head is restrained even though a command to move the head is generated (as measured by neck torque). Accordingly, in Figure $8 C$, we use the term "expected consequence of head command" to refer to the predicted sensory response and to not exclude either possibility.

Two important criteria for a putative site of matching are that the structure receive inputs related to both (1) neck proprioceptive information and (2) neck motor efference copy or, alternatively, an estimate of the expected sensory feedback. A likely candidate would be one or more of the structures in the cerebellum involved in vestibular processing (e.g., nodulus-uvula, flocculas, and fastigial nucleus). For example, the nodulus-avula region receives neck proprioceptive input via the central cervical nucleus (CCN) (Voogd et al., 1996) and direct inputs from the nucleus reticularis gigantocellularis. This latter structure receives direct projections from cortical structures involved in producing head and neck movement (Alstermark et al., 1992a,b). In turn, vestibulospinal neurons, which are thought to be the VO neurons of the present study (Wilson et al., 1990; Boyle et al., 1996; Gdowski and McCrea, 1999), receive direct inputs from the nodulus-uvula (Xiong and Matsushita, 2000). Taken together, these results make the cerebellar nodulus-uvula a strong candidate site. A precedent for this idea comes from work in electric fish, in which it has been proposed that a similar sensory-motor matching occurs in the cerebellum-like electrosensory lobes (Bell et al., 1999; Bell, 2001). Moreover, functional magnetic resonance imaging studies have suggested that the cerebellum serves a similar role in the suppression of tactile stimulation during self-produced tickle (Blakemore et al., 1998, 1999).

The CCN is a second possible candidate site. The CCN is located in the medial part of lamina VII of the spinal cord, extending from C1 to C4 (Rexed, 1954), and receives significant projections from neck muscle spindle afferents (Hirai et al., 1984a; Thompson et al., 1996). In decerebrate and anesthetized animals, CCN neurons respond to passive activation of neck proprioceptors (Hirai et al., 1984a; Hongo et al., 1988; Thompson et al., 1996). Moreover, the CCN sends projections to the vestibular nuclei (Carleton and Carpenter, 1983; Hirai et al., 1984b; McKelvey-Briggs et al., 1989; Sato et al., 1997). However, it remains to be determined in the alert animal whether an efference copy of the neck motor command or an estimate of the expected afference signal is available to these neurons.

The vestibular nuclei also receive direct projections from at least six cortical regions (Akbarian et al., 1993, 1994; Guldin et al., 1993), some of which are involved in the control of head movements, encode neck proprioceptive signals, or both (for review, see Fukushima, 1997). However, response latencies to electrical stimulation are most often longer than would be expected for a monosynaptic projection (Fukushima et al., 1984; Troiani et al., 1993; Wilson et al., 1999). Thus, direct inputs from these areas are most likely to play a relatively minor role in modulating vestibular neuron responses.

\section{Functional implications}

VO neurons activate the neck musculature via direct projections to the ventromedial funiculus of segments $\mathrm{C} 1$ and $\mathrm{C} 2$ of the spinal cord (Boyle and Pompeiano, 1981; Wilson et al., 1990; Gdowski and McCrea 1999). Thus, it has been proposed that they function to mediate the vestibulocollic reflex (VCR). Indeed, their reduced sensitivity during active head movements is consistent with this role because the VCR functions to stabilize the head in space via activation of the neck musculature; during voluntary head-on-body movements, the VCR would be counterproductive. Moreover, VO neurons continue to faithfully encode information about passive head rotations that occur during the execution of voluntary movements (Boyle et al., 1996; McCrea et al., 1999; Roy and Cullen, 2001). Accordingly, as we explore our environment, these neurons would selectively only generate a VCR in response to externally applied head movements.

An even more intriguing implication of the selective gating observed in vestibular nuclei is that it must be incorporated into our understanding of higher-level processing of vestibular information. For example, VO neurons are most likely reciprocally interconnected with the nodulus-uvula of the cerebellum, which in turn transforms head-referenced movement information into an gravity-referenced coordinate frame (Wylie et al., 1994; Angelaki and Hess, 1995; Wearne et al., 1998). Moreover, vestibular projections to the hippocampus are of considerable interest because this structure produces an estimate of current orientation for navigation (O'Keefe and Nadel, 1979). It is commonly thought that on-line integration of the head velocity signal generated by the vestibular nuclei is required for the estimation of head direction by the hippocampal system (for review, see Sharp et al., 2001; Wiener et al., 2002). In addition, regions of the cortex and thalamus that receive inputs from the vestibular nuclei are not sensitive to eye movements (Magnin and Fuchs, 1977; Buttner and Lang, 1979; Grüsser et al., 1990), suggesting that the vestibular information could arrive via VO neurons. Future studies are required to understand how the gated information that is encoded in the vestibular nuclei is used by these higher-order structures to provide perceptual stability during natural behaviors.

\section{References}

Akbarian S, Grüsser O-J, Guldin WO (1993) Corticofugal projections to the vestibular nuclei in squirrel monkeys: further evidence of multiple cortical vestibular fields. J Comp Neurol 332:89-104.

Akbarian S, Grüsser O-J, Guldin WO (1994) Corticofugal connections between the cerebral cortex and brainstem vestibular nuclei in the macaque monkey. J Comp Neurol 339:421-437.

Alstermark B, Pinter MJ, Sasaki S (1992a) Descending pathways mediating disynaptic excitation of dorsal neck motoneurons in the cat: facilitatory interactions. Neurosci Res 15:32-41.

Alstermark B, Pinter MJ, Sasaki S (1992b) Descending pathways mediating disynaptic excitation of dorsal neck motoneurons in the cat: brain stem relay. Neurosci Res 15:42-57.

Anastasopoulos D, Mergner T (1982) Canal-neck interaction in vestibular nuclear neurons of the cats. Exp Brain Res 46:269-280.

André-Deshays C, Revel M, Berthoz A (1991) Eye-head coupling in humans. II. Phasic components. Exp Brain Res 84:359-366.

Angelaki DE, Hess BJ (1995) M. Inertial representation of angular motion in the vestibular system of rhesus monkeys. II. Otolith-controlled transformation that depends on an intact cerebellar nodulus. J Neurophysiol 73:1729-1751.

Bell CC (2001) Memory-based expectations in electrosensory systems. Curr Opin Neurobiol 11:481-487.

Bell CC, Han VZ, Sugawara Y, Grant K (1999) Synaptic plasticity in the mormyrid electrosensory lobe. J Exp Biol 202:1339-1347. 
Bizzi E, Kalil RE, Tagliasco V (1971) Eye-head coordination in monkeys: evidence for centrally patterned organization. Science 173:452-454.

Blakemore SJ, Wolpert DM, Frith CD (1998) Central cancellation of selfproduced tickle sensation. Nat Neurosci 1:635-640.

Blakemore SJ, Wolpert DM, Frith CD (1999) The cerebellum contributes to somatosensory cortical activity during self-produced tactile stimulation. Neuroimage 10:448-459.

Boyle R, Pompeiano O (1981) Responses of vestibulospinal neurons to sinusoidal rotation of the neck. J Neurophysiol 44:633-649.

Boyle R, Belton T, McCrea RA (1996) Responses of identified vestibulospinal neurons to voluntary eye and head movements in the squirrel monkey. Ann NY Acad Sci 781:244-263.

Buttner U, Lang W (1979) The vestibulocortical pathway: neurophysiological and anatomical studies in the monkey. Prog Brain Res 50:581-588.

Carleton SC, Carpenter MB (1983) Afferent and efferent connections of the medial, inferior and lateral vestibular nuclei in the cat and monkey. Brain Res 278:29-51.

Cullen KE, Guitton D (1997) Analysis of primate IBN spike trains using system identification techniques. II. Relationship to gaze, eye, and head movement dynamics during head-free gaze shifts. J Neurophysiol 78:3283-3306.

Cullen KE, McCrea RA (1993) Firing behavior of brain stem neurons during voluntary cancellation of the horizontal vestibulo-ocular reflex. I. secondary vestibular neurons. J Neurophysiol 70:828-843.

Cullen KE, Minor LB (2002) Semicircular canal afferents similarly encode active and passive head rotation: implications for the role of vestibular efference. J Neurosci 22:RC226(1-7).

Cullen KE, Rey CG, Guitton D, Galiana HL (1996) The use of system identification techniques in the analysis of oculomotor burst neuron spike train dynamics. J Comp Neurosci 3:347-368.

Decety J (1996) Neural representation for action. Rev Neurosci 7:285-297.

Fuchs AF, Kimm J (1975) Unit activity in vestibular nucleus of the alert monkey during horizontal angular acceleration and eye movement. J Neurophysiol 38:1140-1161.

Fuchs AF, Robinson DA (1966) A method for measuring horizontal and vertical eye movements in the monkey. J Physiol (Lond) 191:609-631.

Fukushima K (1997) Corticovestibular interactions: anatomy, electrophysiology, and functional considerations. Exp Brain Res 117:1-16.

Fukushima K, Takahashi K, Ohno M, Kato M (1984) Responses of cat vestibular neurons to stimulation of the frontal cortex. Exp Brain Res 56:275-278.

Gdowski GT, McCrea RA (1999) Integration of vestibular and head movement signals in the vestibular nuclei during whole-body rotation. J Neurophysiol 81:436-449.

Grüsser OJ, Pause M, Schreiter U (1990) Localization and responses of neurones in the parieto-insular vestibular cortex of awake monkeys (Macaca fascicularis). J Physiol (Lond) 430:537-557.

Guldin WO, Mirring S, Grüsser O-J (1993) Connections from the neocortex to the vestibular brainstem nuclei in the common marmoset. NeuroReport 5:113-116.

Hayes AV, Richmond BJ, Optican LM (1982) A UNIX-based multiple process system for real-time data acquisition and control. In: Western Electronic Show and Conference Proceedings, Vol 2, pp 1-10. Institute of Electrical and Electronics Engineers.

Hirai N, Hongo T, Sasaki S (1984a) A physiological study of identification, axonal course and cerebellar projection of spinocerebellar tract cells in the central cervical nucleus of the cat. Exp Brain Res 55:272-285.

Hirai N, Hongo T, Sasaki S, Yamashita M, Yoshida K (1984b) Neck muscle afferent input to spinocerebellar tract cells of the central cervical nucleus in the cat. Exp Brain Res 55:286-300.

Hongo T, Kitama T, Yoshida K (1988) Integration of vestibular and neck afferent signals in the central cervical nucleus. Prog Brain Res 76:155-162.

Jeannerod M (1988) The neural and behavioral organization of goaldirected movements. Oxford: Oxford UP.

Kasper J, Wilson VJ, Yamagata Y, Yates BJ (1989) Neck muscle spindle activity in the decerebrate, unparalyzed cat: dynamics and influence of vestibular stimulation. J Neurophysiol 62:917-923.

Keller EL, Kamath BY (1975) Characteristics of head rotation and eye movementrelated neurons in alert monkey vestibular nucleus. Brain Res 100:182-187.

Lestienne F, Vidal PP, Berthoz A (1984) Gaze changing behaviour in head restrained monkey. Exp Brain Res 53:349-356.

Lisberger SG, Westbrook LE (1985) Properties of visual inputs that initiate horizontal smooth pursuit eye movements in monkeys. J Neurosci 5:1662-1673.

Magnin M, Fuchs AF (1977) Discharge properties of neurons in the monkey thalamus tested with angular acceleration, eye movement and visual stimuli. Exp Brain Res 28:293-299.

McCrea RA, Gdowski GT, Boyle R, Belton T (1999) Firing behavior of vestibular neurons during active and passive head movements: vestibulo-spinal and other non-eye-movement related neurons. J Neurophysiol 82:416-428.

McKelvey-Briggs DK, Saint-Cyr JA, Spence SJ, Partlow GD (1989) A reinvestigation of the spinovestibular projection in the cat using axonal transport techniques. Anat Embryol 180:281-291.

O’Keefe J, Nadel L (1979) The hippocampus as a cognitive map. Behav Brain Res 34:171-175.

Rexed B (1954) A cytoarchitectonic atlas of the spinal cord of the cat. J Comp Neurol 100:97-379.

Richmond FJR, Abrahams VC (1979) Physiological properties of muscle spindles in dorsal neck muscles of the cat. J Neurophysiol 42:604-617.

Richmond FJR, Bakker DA, Stacey MJ (1988) The sensorium: receptors of neck muscles and joints. In: Control of head movement (Peterson BW, Richmond FJ, eds), pp 49-62. New York: Oxford UP.

Roy JE, Cullen KE (2001) Selective processing of vestibular reafference during self-generated head motion. J Neurosci 21:2131-2142.

Sato H, Ohkawa T, Uchino Y, Wilson VJ (1997) Excitatory connections between neurons of the central cervical nucleus and vestibular neurons in the cat. Exp Brain Res 115:381-386.

Scudder CA, Fuchs AF (1992) Physiological and behavioural identification of vestibular nucleus neurons mediating the horizontal vestibuloocular reflex in trained rhesus monkeys. J. Neurophysiol 68:244-264.

Sharp PE, Tinkelman A, Cho J (2001) Angular velocity and head direction signals recorded from the dorsal tegmental nucleus of Gudden in the rat: implications for path integration in the head direction cell circuit. Behav Neurosci 115:571-588.

Tangorra JL, Jones LA, Hunter IW (2003) Dynamics of the human headneck system in the horizontal plane: joint properties with respect to a static torque. Ann Biomed Eng 31:606-620.

Thompson DB, Isu N, Wilson VJ (1996) Responses of neurons of the cat central cervical nucleus to natural neck and vestibular stimulation. J Neurophysiol 76:2786-2789.

Tomlinson RD, Robinson DA (1984) Signals in vestibular nucleus mediating vertical eye movements in the monkey. J Neurophysiol 51:1121-1136.

Troiani D, Draicchio F, Bonci A, Zanmoni B (1993) Responses of vestibular neurons to stimulation of cortical sensorimotor areas in the cat. Arch Ital Biol 131:137-146.

Vidal PP, Roucoux A, Berthoz A (1982) Horizontal eye position-related activity in neck muscles of the alert cat. Exp Brain Res 46:448-453.

von Holst E, Mittelstaedt H (1950) Das reafferenzprinzip. Naturwissenschaften 37:464-476.

Vallbo AB (1981) Basic patterns of muscle spindle discharge in man. In: Muscle receptors and movement (Taylor A, Prochazka A, eds), pp 219 228. London: Macmillan.

Voogd J, Gerrits M, Ruigrok JH (1996) Organization of the vestibulocerebellum. Ann NY Acad Sci 781:553-579.

Wearne S, Raphan T, Cohen B (1998) Control of spatial orientation of the angular vestibuloocular reflex by the nodulus and uvula. J Neurophysiol 79:2690-2715.

Wiener SI, Berthoz A, Zugaro MB (2002) Multisensory processing in the elaboration of place and head direction responses by limbic system neurons. Cognit Brain Res 14:75-90.

Wilson VJ (1991) Vestibulospinal and neck reflexes: interaction in the vestibular nuclei. Ach Ital Biol 129:43-52.

Wilson VJ, Yamagata Y, Yates BJ, Schor RH, Nonaka S (1990) Response of vestibular neurons to head rotations in vertical planes. III. response of vestibulocollic neurons to vestibular and neck stimulation. J Neurophysiol 64:1695-1703.

Wilson VJ, Zarzecki P, Schor RH, Isu N, Rose PK, Sato H, Thomson DB, Umezaki T (1999) Cortical influences on the vestibular nuclei of the cat. Exp Brain Res 125:1-13.

Wolpert DM, Ghahramani Z, Jordan M (1995) An internal model for sensorimotor integration. Science 269:1880-1882.

Wylie DR, De Zeeuw CI, Digiorgi PL, Simpson JI (1994) Projections of individual Purkinje cells of identified zones in the ventral nodulus to the vestibular and cerebellar nuclei in the rabbit. J Comp Neurol 349:448-463.

Xiong G, Matsushita M (2000) Connections of Purkinje cell axons of lobule $\mathrm{X}$ with vestibulospinal neurons projecting to the cervical cord in the rat. Exp Brain Res 131:491-499. 